\title{
A novel streptococcal surface protease promotes virulence, resistance to opsonophagocytosis, and cleavage of human fibrinogen
}

\author{
Theresa O. Harris, ${ }^{1}$ Daniel W. Shelver, ${ }^{1}$ John F. Bohnsack, ${ }^{2}$ and Craig E. Rubens ${ }^{1}$ \\ ${ }^{1}$ Division of Infectious Disease, Children's Hospital and Regional Medical Center, and University of Washington, \\ Seattle, Washington, USA \\ ${ }^{2}$ Department of Pediatrics and Department of Pathology, University of Utah School of Medicine, Salt Lake City, Utah, USA
}

Group B streptococcus (GBS) is an important human pathogen. In this study, we sought to identify mechanisms that may protect GBS from host defenses in addition to its capsular polysaccharide. A gene encoding a cell-surface-associated protein $(c s p A)$ was characterized from a highly virulent type III GBS isolate, $\mathrm{COH} 1$. Its sequence indicated that it is a subtilisin-like extracellular serine protease homologous to streptococcal C5a peptidases and caseinases of lactic acid bacteria. The wild-type strain cleaved the $\alpha$ chain of human fibrinogen, whereas a cspA mutant, TOH121, was unable to cleave fibrinogen. We observed aggregated material when $\mathrm{COH} 1$ was incubated with fibrinogen but not when the mutant strain was treated similarly. This suggested that the product(s) of fibrinogen cleavage have strong adhesive properties and may be similar to fibrin. The $\operatorname{csp} A$ gene was present among representative clinical isolates from all nine capsular serotypes, as revealed by Southern blotting. A $\operatorname{csp} A^{-}$mutant was ten times less virulent in a neonatal rat sepsis model of GBS infections, as measured by $\mathrm{LD}_{50}$ analysis. In addition, the $c s p A^{-}$mutant was significantly more sensitive than the wildtype strain to opsonophagocytic killing by human neutrophils in vitro. Taken together, the results suggest that cleavage of fibrinogen by CspA may increase the lethality of GBS infection, potentially by protecting the bacterium from opsonophagocytic killing.

J. Clin. Invest. 111:61-70 (2003). doi:10.1172/JCI200316270.

\section{Introduction}

Group B streptococcus (GBS) is a major cause of serious neonatal bacterial infections (1) and is the most common cause of sepsis and pneumonia in newborns. GBS is also a significant cause of postpartum endometritis. In addition, recent data implicate GBS as an increasingly important cause of invasive infections in adults, especially among the immunocompromised (2).

Bacterial pathogens have evolved a diverse array of defenses to combat the innate immune system, which is an important first line of defense against bacterial infections in the nonimmune host. Complement cells, as well as phagocytic cells such as polymorphonuclear leukocytes (PMNs) and macrophages, play major roles in innate immunity. The antiphagocytic mechanisms

Received for publication June 24, 2002, and accepted in revised form September 17, 2002.

Address correspondence to: Craig E. Rubens,

4800 Sand Point Way NE, Seattle, Washington 98105, USA. Phone: (206) 526-2073; Fax: (206) 527-3890;

E-mail: cruben@chmc.org.

Theresa O. Harris and Daniel Shelver contributed equally to this work.

Conflict of interest: The authors have declared that no conflict of interest exists.

Nonstandard abbreviations used: group B streptococcus (GBS); cell-surface-associated protein (CspA); polymorphonuclear leukocytes (PMNs); group A streptococcus (GAS); Todd-Hewitt broth (THB); matrix-assisted laser desorption/ionization time-of-flight mass spectrometry (MALDI-TOF MS); cell envelope-associated protease (CEP). of a number of Gram-negative bacteria such as Yersiniae and Pseudomonas aeruginosa (3) are well characterized. However, much remains to be understood about how Gram-positive pathogens evade phagocytic mechanisms. Streptococcus pneumoniae strains produce polysaccharide capsules (4) that allow the bacterium to resist complement deposition in the absence of typespecific capsule antibodies. In addition, S. pneumoniae strains produce a $\mathrm{C} 3$ binding protein that may function in the evasion of complement-mediated host defense (5). Enterococcus faecalis, a major cause of hospital-acquired infections, has recently been reported to synthesize a capsular polysaccharide that provides resistance to opsonophagocytic killing (6). Strains of group A streptococcus (GAS) use numerous mechanisms to evade this immune pathway, including the $\mathrm{M}$ protein (7) and the hyaluronic acid capsule (8).

The GBS capsule is the most well-defined virulence factor of GBS. The capsule protects GBS from opsonization by C3 through inhibition of the alternative complement pathway in the absence of type-specific capsule antibodies (9). However, GBS may express additional factors that allow it to resist opsonophagocytosis. A recent report indicated that the surface-localized streptococcal $\beta$ protein binds human complement factor $\mathrm{H}$ and that the GBS-factor $\mathrm{H}$ complex retains its ability to downregulate complement activation (10).

In this study, we identify $\operatorname{csp} A$, a novel gene encoding a surface-localized, serine protease-like protein that promotes GBS survival through evasion of opsonophago- 
cytosis. CspA shows homology to a family of proteases that include C5a proteases of pathogenic streptococci (11) as well as caseinases expressed by nonpathogenic Gram-positive cocci (12). Surprisingly, we observed that CspA does not have enzymatic activity against C5a in vitro and the presence of the $\operatorname{csp} A$ gene was not required for casein degradation. However, the $\operatorname{csp} A$ gene was required for GBS cleavage of human fibrinogen, which indicates that CspA is active as a protease. Mutants that failed to express $\operatorname{csp} A$ displayed a significantly decreased GBS virulence in a neonatal rat model of infection and displayed increased sensitivity to opsonophagocytosis. Our findings provide evidence that CspA is a novel, surface-localized protease that plays an important role in GBS pathogenesis as an antiphagocytic surface factor.

\section{Methods}

Bacterial strains. $\mathrm{COH} 1$ is a highly encapsulated type III GBS strain, originally isolated from the blood of a septic newborn (13). Other GBS clinical strains used in this work included type 1a strains B523 (14), A909 (14), and ChanS5; type Ib strains DK14, DK15, and 80-481; type II strains 78-471 (15) and DK23 (14); type III strains COH31 (9), D136C (14), and M781 (14); type IV strain CNCTC1/82 (14); type V strains B201 and CNCTC 10/84 (14); type VI strain NT6 (14); type VII strain 87-603; and type VIII strain JM9 (kindly provided by Pat Ferrieri, University of Minnesota, Minneapolis, Minnesota, USA). COH1-13 is an acapsular Tn916 $\triangle \mathrm{E}$ mutant of $\mathrm{COH} 1$ (16).

Media, chemicals, and culture of bacterial strains. Escherichia coli and GBS were grown in Luria broth and ToddHewitt broth (THB), respectively. Concentrations of antibiotics for selection included ampicillin (Amp, 75 $\mu \mathrm{g} / \mathrm{ml}$ ), erythromycin (Erm, $400 \mu \mathrm{g} / \mathrm{ml}$ for E. coli and 10 $\mu \mathrm{g} / \mathrm{ml}$ for GBS), or chloramphenicol (Cam, $10 \mu \mathrm{g} / \mathrm{ml}$ ). For the culture of GBS in human plasma, plasma was obtained from healthy human donors who provided consent. GBS was grown to $\mathrm{OD}_{600}$ of 0.6 in THB, washed twice in an equal volume of PBS, and resuspended in plasma at a concentration of approximately $1000 \mathrm{CFU} / \mathrm{ml}$. Growth was then monitored over a 6hour period, duplicate dilutions were plated, and doubling time was calculated.

DNA and RNA methods. Standard procedures for cloning, sequencing, Southern blotting, Northern blotting, and PCR amplification were used (17). RNA was isolated by the method of Yim and Rubens (18). Antisense digoxigenin-labeled probes were used for Northern blot procedures as recommended by the manufacturer (Roche Molecular Biochemicals, Indianapolis, Indiana, USA).

Identification and cloning of the cspA locus. A portion of the $\operatorname{csp} A$ open reading frame was originally isolated by analysis of the transposon insertion site from a Tn916 $\mathrm{E}$ mutant of strain $\mathrm{COH} 1$ and was used as a probe to clone the entire $\operatorname{csp} A$ gene. Overlapping $C l a \mathrm{I}$ and $X b a \mathrm{I}$ restriction fragments (Figure 1) that bear $\operatorname{csp} A$ were identified by Southern analysis of $\mathrm{COH} 1$ genomic DNA and cloned into pBSKS- (Stratagene, La Jolla,
California, USA) using standard techniques. E. coli DH5 $\alpha$ clones harboring the desired GBS inserts were identified by colony blots using the probe mentioned above. Clones containing either a ClaI fragment (TOH37 containing plasmid pTH2) or an XbaI fragment (TOH50 containing plasmid pTH5) were further analyzed. The cloned cspA gene present on plasmid pTH5 contains a spontaneous mutation, in comparison with the chromosomal $\operatorname{csp} A$ sequence of the wildtype isogenic strain, $\mathrm{COH} 1$; this mutation is predicted to terminate translation prematurely at Leu-1121. The sequences described in this work have been deposited in GenBank under accession number AY162834.

Construction of a cspA::erm mutation in GBS. To perform allelic replacement mutagenesis of $\operatorname{csp} A$, we subcloned $\operatorname{csp} A$ to pVE6007, a broad-host-range plasmid that replicates at $28^{\circ} \mathrm{C}$ but not at $37^{\circ} \mathrm{C}(19)$. A 5.4-kb PCR product of cspA was amplified from $\mathrm{COH} 1$ genomic DNA, digested with BamHI and $\mathrm{XbaI}$, and cloned into Bam HI/XbaI-digested pVE6007, generating intermediate plasmid pTH19. Approximately $3.6 \mathrm{~kb}$ of $\operatorname{csp} A$ sequence (corresponding to CspA peptide residues 323-1536) flanked by HindIII sites was subsequently replaced with the $\mathrm{erm}^{\mathrm{r}}$ gene from pCER1000 (20). The final construct, $\mathrm{pTH} 21$, was transformed into $\mathrm{COH} 1$ as described (21). A strain that bears a replacement of $\operatorname{csp} A$ with the $\mathrm{erm}^{\mathrm{r}}$ element was obtained by curing the plasmid, as described by Yim and Rubens (22), and was designated TOH121. The presence of the desired mutation on the chromosome of TOH121 was verified by Southern blotting.

Phenotypic and $L D_{50}$ virulence assays. Analysis of cellassociated type III GBS capsule (23), beta-hemolysin, CAMP factor (a factor that destabilizes and lyses erythrocyte membranes) (24), hyaluronidase (25), and hippuricase expression was performed as described (26). Caseinase activity was evaluated by several means. First, caseinase activity was measured by growing GBS overnight on $\mathrm{THB}+5 \%$ milk agar plates. Second, caseinase activity was measured by incubating GBS culture supernatants, mutanolysin extracts, or whole cells with purified, protease assay-grade casein (SigmaAldrich, St. Louis, Missouri, USA). Adherence and invasion assays of A549 cell monolayers (type II lung epithelial cells) were performed as described (27). C5a protease activity of individual strains was determined by the ability of GBS to inhibit C5a-stimulated adherence of human PMNs to gelatin-coated tissue-culture wells as described (28). The virulence of isogenic $\operatorname{csp} A^{+/-}$ strains was compared using a neonatal rat model of lethal GBS infection by $\mathrm{LD}_{50}$ analysis as described previously (29); statistical analysis for the $\mathrm{LD}_{50}$ data was performed with the Wilcoxon matched-pair signedranks test. All animals were maintained according to institutional, state, and federal guidelines.

Recombinant CspA-GST fusion-protein production and generation of CspA antibody. A CspA-GST fusion protein was constructed for use as an immunogen. A C-terminal portion of CspA that lacks the putative catalytic domain was 
amplified from $\mathrm{COH} 1$ chromosomal DNA with primers TCGGATCCGCTACTGCTCTAGTT and TTAAGTCGACGTAATGATGCCTTGCTCTA, which incorporate BamHI and SalI sites for cloning. Plasmid pGEX-4T-3 (AmershamPharmacia Biosciences, Piscataway, New Jersey, USA) was digested with BamHI and SalI and ligated to the PCR product, which was also digested with BamHI and SalI. The CspA-GST fusion protein formed inclusion bodies; after solubilization and SDS-PAGE, CspA was excised from polyacrylamide gels and fragmented as described (30). This preparation was used to immunize a New Zealand white rabbit previously shown to lack antibody to the fusion protein.

Western blot analysis of CspA. CspA was released from the periplasmic space of TOH50 ( $E$. coli DH5 $\alpha$ harboring plasmid pTH5) by an osmotic shock procedure (31). GBS cell-surface-associated proteins were extracted by treatment with mutanolysin and subjected to SDSPAGE (32). Proteins were transferred to Immobilon-P (Millipore Inc., Bedford, Massachusetts, USA); primary antibody and secondary horseradish peroxidase-conjugated antibody were used at dilutions of 1:500 and 1:1000, respectively. The SuperSignal reagent (Pierce Biotechnology Inc., Rockford, Illinois, USA) was utilized for the chemiluminescent detection of Western blots.

Fibrinogen degradation assays. Purified human fibrinogen, depleted of fibronectin and plasminogen, was purchased from Enzyme Research Laboratories (South Bend, Indiana, USA). To assay fibrinogen degradation, GBS strains COH1 and TOH121 were grown to stationary phase, washed once in PBS, concentrated 20-fold, and resuspended in PBS. Fibrinogen was then added at a concentration of $0.63 \mathrm{mg} / \mathrm{ml}$. The fibrinogen/cell suspension was incubated with slow rotation at $37^{\circ} \mathrm{C}$. After 16 hours, GBS cells were removed by centrifugation. The supernatant was analyzed by SDS-PAGE using large format $(18.5 \times 20 \mathrm{~cm}), 10 \%$ acrylamide gels to resolve the species of the fibrinogen $\alpha$ chain.

Matrix-assisted laser desorption/ionization time-of-flight mass spectrometry offibrinogen $\alpha$ species. The identity of the two $\alpha$-chain species was confirmed by MALDI-TOF MS (matrix-assisted laser desorption/ionization time-offlight mass spectrometry). The fibrinogen $\alpha$ chains were separated by SDS-PAGE as described above, and the upper- and lower-migrating species were excised from the Coomassie-stained gel. The gel slices were destained overnight in 50\% methanol. The methanol was removed, acetonitrile was added to cover the gel slices, and the mixture was incubated for 10 minutes. The acetonitrile was evaporated under vacuum. Trypsin (sequencing grade; Promega, Madison, Wisconsin, USA) was added to the dehydrated gel fragments, incubated for 45 minutes at $4^{\circ} \mathrm{C}$, and then incubated overnight at $37^{\circ} \mathrm{C}$. After overnight incubation, the trypsin solution was removed, and the gel slices were extracted twice with $200 \mu \mathrm{l}$ of $5 \%$ formic acid and 50\% acetonitrile. The trypsin solution was pooled with the extraction solution and evaporated under vacuum. Ten microliters of $5 \%$ acetonitrile and $0.5 \%$ acetic acid was added, and 0.6 $\mu \mathrm{l}$ was spotted on the target. After this, mass spectra were acquired using a BIFLEX III mass spectrometer (Bruker, Billerica, Massachusetts, USA).

Opsonophagocytosis assays. Opsonophagocytosis assays were performed using serum and neutrophils that were obtained with consent from nonimmune humans, as previously described (33). All assays were performed in triplicate, and controls included samples with heat-inactivated sera $\left(56^{\circ} \mathrm{C}\right.$ for 30 minutes $)$ and without PMNs.

\section{Results}

It is likely that many factors contribute to the virulence of GBS (29). In order to identify novel virulence factors, we performed a screen to identify transposon mutants with reduced virulence in GBS. In the process of screening Tn916 $\Delta \mathrm{E}$ mutants of the highly virulent type III GBS isolate, COH1, we identified a gene with homology to cell-surface-associated proteases. The open reading frame, which we designated $\operatorname{csp} A$ (Figure 1), encodes a 1,571-amino acid protein and displays homology to several extracellular serine proteases from the subtilase family. The greatest similarity of CspA (51.2\% identity and $58.3 \%$ similarity) was to PrtS, which is an extracellular caseinase produced by $S$. thermophilus (12). The next highest similarity was to a putative extracellular protease (39\% identity and 55\% similarity) from the GAS genome sequence (34). The third highest similarity $(45 \%$ similarity and $36 \%$ identity) was to ScpA (35) and ScpB

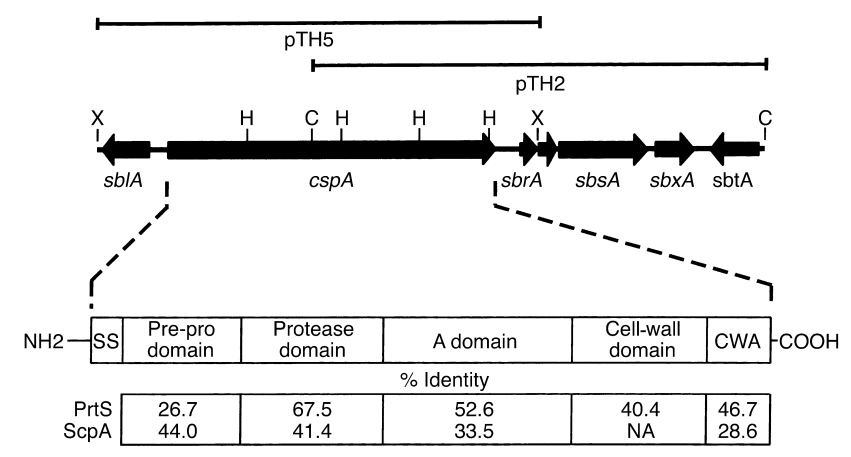

\section{Figure 1}

Map of the cspA region in type III GBS. Arrows depict the identified open reading frames and their direction of transcription. Plasmid inserts used to sequence the locus are depicted above the map. The terminal $5^{\prime}$ and $3^{\prime}$ Hind III sites at positions 2342 and 5983 were used to replace the internal cspA coding sequence with $\mathrm{erm}^{\mathrm{r}}$. The $s b x A$ gene encodes a product of 202 amino acids with no homology to characterized proteins. The 337 -amino acid product of sbtA shares significant homology with the $\mathrm{C}$-terminal coding region of numerous tRNA synthetases. Shown below the open reading frame map are protein motifs identified in the predicted sequence of CspA (36): signal sequence (residues 1-35), pre-pro domain (residues 36-143), protease domain (residues 144-638), A domain (residues 639-1076), cell-wall spacer domain (residues 1077-1535) and cell-wall anchor domain (residues 1536-1571). An RGD motif (53) is present in the protease domain and position 446 . The function of the A domain is unknown, but it may be involved in regulation or substrate specificity of the protease domain (36). C, Clal; X, Xbal; H, HindIII; SS, signal sequence; CWA, cell-wall anchor domain. 


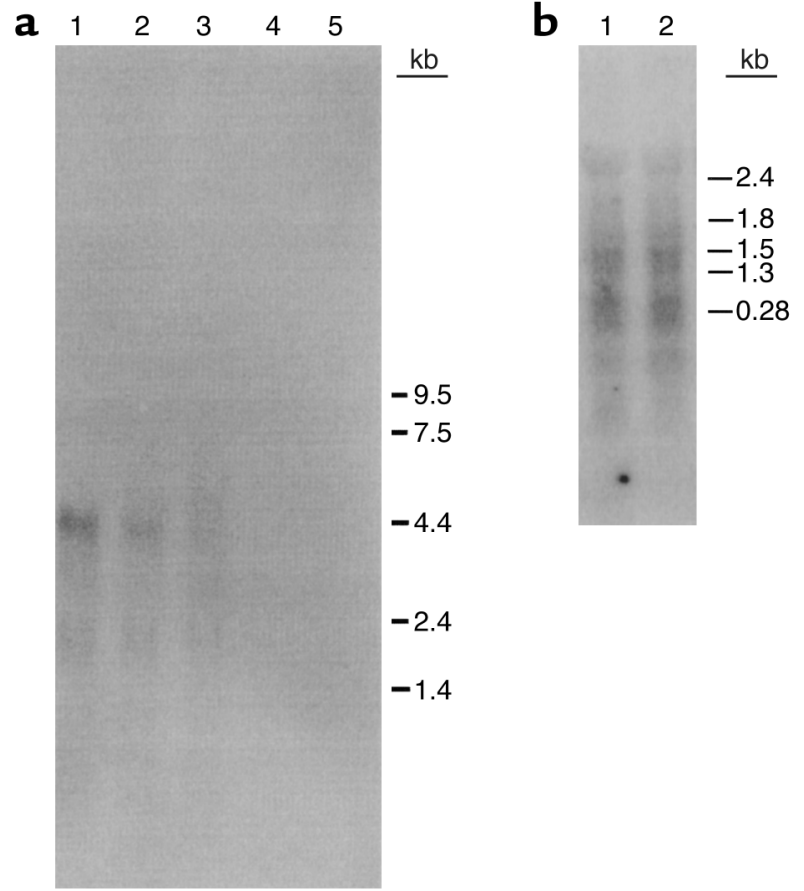

\section{Figure 2}

Northern blot analysis of cspA expression in $\mathrm{COH} 1$ and TOH121. Standard Northern blots were performed using equivalent amounts of RNA $(5 \mu \mathrm{g})$ from the indicated strains. (a) Blot developed using $\operatorname{csp} A$ probe. Lane $1, \mathrm{COH} 1$ at $\mathrm{OD}_{600}=0.3$ in $\mathrm{THB}$; lane $2, \mathrm{COH} 1$ at $\mathrm{OD}=0.6$; lane $3, \mathrm{COH} 1$ at $\mathrm{OD}=1.7$; lane $4, \mathrm{TOH} 121$ at $\mathrm{OD}=0.3$; lane $5, \mathrm{TOH} 121$ at $\mathrm{OD}=1.7$. (b) Blot developed using sbrA probe. Lane $1, \mathrm{COH} 1$ at $\mathrm{OD}=0.6$; lane $2, \mathrm{TOH} 121$ at $\mathrm{OD}=0.6$. Migration of RNA size standards is indicated (in $\mathrm{kb}$ ) on the right.

(28), which participate in the proteolytic inactivation of the chemotactic factor C5a in GAS and GBS, respectively. In addition, CspA was similar to several caseinases of lactic acid bacteria (27\% identity and $36 \%$ similarity) encoded by the $\operatorname{prtB}$, $\operatorname{prtH}$, and $\operatorname{prtP}$ genes (36).

The sequence of CspA indicates that it shares the functional and structural domains of the cell envelope-associated protease (CEP) family (36). The CEPs are a subfamily of subtilisin-like serine proteases that are found in a wide variety of bacteria. An excellent comprehensive review of the properties of the CEP protein family may be found in Siezen (36). The motifs required for the catalytic function of this family of serine proteases are present in CspA at residues 168-179 (aspartic acid motif, VAIISDGLNTNH), 237-248 (histidine motif, HGMHVTSIATA) and 565-575 (serine motif, GTSMASPHVAG); this suggests that CspA functions as a protease.

A general characteristic of caseinases is that they are synthesized as pre-pro enzymes and, after translocation across the cell membrane, are activated by autocatalytic cleavage of a pro-peptide sequence (36). A putative pre-pro domain, spanning residues $1-143$, was identified in CspA (36), implying that CspA may also be synthesized as a pre-pro enzyme and may similarly undergo autocatalytic maturation.

Inactivation of $\operatorname{csp} \mathrm{A}$ and $\operatorname{scpB}$. To facilitate the functional analysis of $\mathrm{CspA}$, allelic replacement mutagenesis of $\operatorname{csp} A$ was performed. A plasmid bearing a $\operatorname{csp} A::$ erm allele was created by deleting a portion of $\operatorname{csp} A$ and replacing it with an erythromycin resistance gene (see Methods). This construct was used to replace the wildtype $\operatorname{csp} A$ allele of $\mathrm{COH} 1$ (a highly virulent, type III GBS clinical isolate), as detailed in the Methods. A single clone, TOH121, was selected for further study, and the presence of the cspA::erm mutation on the chromosome of this strain was verified by Southern blotting. To provide controls for evaluating GBS C5a protease activity (see below), a mutant bearing a kanamycin resistance element insertion in the GBS C5a peptidase gene, $s c p B$, was also constructed in the $\mathrm{COH} 1$ genetic background and was designated TOH97. A double mutant bearing both the $\operatorname{csp} A:: e r m$ and the $\operatorname{sp} B$ :::kan mutations was constructed and designated TOH144. Southern blotting was also used to confirm the presence of the desired mutation for the two $s c p B:: k a n$ mutants (data not shown).

The cspA gene is transcribed as a monocistronic operon. We characterized the transcriptional organization of the region proximal to $\operatorname{csp} A$. Examination of the DNA region next to $\operatorname{cs} p A$ revealed open reading frames located both $5^{\prime}$ and $3^{\prime}$ of $\operatorname{csp} A$ (Figure 1). A gene designated sblA (for streptococcus group B lipoprotein) is located $5^{\prime}$ of $\operatorname{csp} A$ and is oriented in the opposite transcriptional direction as cspA. The predicted product shares 33\% identity with AzlC from Deinococcus radiodurans (37); the precise biological function of the AzlC protein is currently unknown (38). Adjacent to $\operatorname{csp} A$ and oriented in the same direction are two putative genes (designated $s b r A$ and $s b s A$ ) that encode products with homology to the response regulator and sensor proteins of two-component regulatory systems (39). A potential promoter sequence is located in the $233 \mathrm{bp}$ of noncoding sequence between $\operatorname{csp} A$ and $s b r A$, suggesting that $s b r A$ and $s b s A$ are transcribed from a promoter that is distinct from that of $\operatorname{csp} A$.

To characterize the transcriptional organization of the cspA region, RNA was isolated from THB-grown $\mathrm{COH} 1$ at different growth stages (early-log, mid-log, and sta-

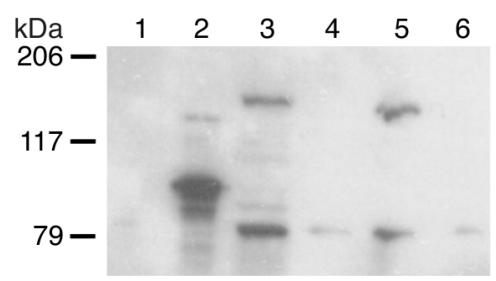

Figure 3

Western blot analysis of CspA expression in E. coli and GBS strains using anti-CspA sera. Lane 1 shows periplasmic extract from $E$. coli $\mathrm{DH} 5 \alpha$ containing pBS (negative control); Lane 2 shows periplasmic extract from E. coli DH5 $\alpha$ containing pTH5 (Xbal fragment bearing cspA in pBSKS-; Stratagene). Note that that the lower migration of CspA in periplasmic extracts is due to a mutation in $\mathrm{PTH} 5$ that prematurely terminates translation (see Methods). Lanes 3-6 show mutanolysinextracted GBS surface proteins from $\mathrm{COH} 1$ (lane 3), $\mathrm{TOH} 121$ (csp $A^{-}$, lane 4), TOH97 (scpB-, lane 5), and $\mathrm{TOH} 144\left(\operatorname{csp} A^{-}, \operatorname{scp} B^{-}\right.$, lane 6). Migration of molecular mass markers (in $\mathrm{kDa}$ ) is indicated on the left. 


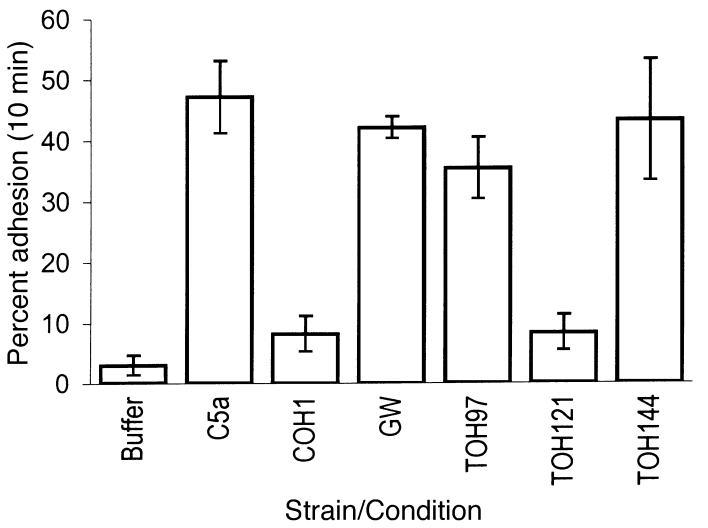

Figure 4

Functional assay for C5a protease activity. Shown is the percent adhesion by human PMNs to gelatin-coated tissue-culture wells after the indicated GBS strains were incubated with recombinant human $\mathrm{C} 5 \mathrm{a}$ (28). As controls, buffer alone (buffer) or $100 \mathrm{ng} / \mathrm{ml}$ of untreated $\mathrm{C} 5 \mathrm{a}$ ( $\mathrm{C5}$ a) were incubated without bacteria before exposure to PMNs. GBS strains that were tested were $\mathrm{COH} 1$ (positive control, $\left.\operatorname{csp} A^{+}, \operatorname{scp} B^{+}\right), G W(32)$ (negative control, a type III GBS strain lacking C5a-ase activity), TOH97 (negative control, $c s p A^{+}, \operatorname{scp} B^{-}$), TOH121 $\left(\operatorname{csp} A^{-}, \operatorname{scp} B^{+}\right)$, and $\mathrm{TOH} 144\left(\operatorname{csp} A^{-}, \operatorname{scp} B^{-}\right)$.

tionary phase) and Northern analysis was performed using a probe internal to the $\operatorname{csp} A$ coding region (Figure 2a). A discrete band corresponding to a transcript size of $4.7 \mathrm{~kb}$ was present in cells at different stages of growth (Figure 2a; lanes 1-3). A control for probe specificity was performed with strain TOH121; no hybridizing material was seen for this strain, as expected. Maximal expression was observed in early-log phase $\left(\mathrm{OD}_{600}=0.3\right.$ ) (Figure $2 \mathrm{a}$, lane 1). Since the coding region of $\operatorname{csp} A$ is approximately $4.7 \mathrm{~kb}$, these observations suggest that $\operatorname{csp} A$ is expressed on a monocistronic transcript.

To confirm our hypothesis that the $\operatorname{csp} A$ transcript is monocistronic, we performed Northern blot analysis on a gene located $3^{\prime}$ to $\operatorname{csp} A$. It was important to verify that the phenotypes (see below) of the cspA mutant, TOH121, were not due to a polar effect of the $\operatorname{csp} A$ mutation onto the adjacent $s b r A$ and $s b s A$ genes (Figure 1). RNA was isolated from $\mathrm{COH} 1$ and TOH 121 in mid$\log$ phase and was hybridized to a probe internal to the coding region of $s b r A$ (see Figure 1). The $s b r A$-hybridizing material ran as a smear at molecular weights that corresponded to less than $2.4 \mathrm{~kb}$. Similar amounts of hybridizing material were observed in both $\mathrm{COH} 1$ and TOH121 (Figure 2b). The cspA and sbrA probes hybridized to distinct transcripts, suggesting that $\operatorname{csp} A$ is transcribed independently from the adjacent genes and that $\operatorname{csp} A$ is transcribed as a monocistron.

CspA is a surface-associated, cell-wall-anchored protein. In addition to predicting that CspA functions as a protease, the sequence of CspA suggests that it is secreted and subsequently anchored to the cell surface. A 35-residue signal peptide within the $\mathrm{N}$-terminal end of CspA was identified using SIGSEQ (40) (Figure 1). A classic C-terminal cell-wall attachment site sequence (LPKTG) characteristic of Gram-positive surface-associated proteins (41) was located at amino acids 1536-1540 (Figure 1). To investigate the hypothesis that CspA is a surface-attached protein, we determined the subcellular localization of CspA by Western blot analysis. Antibody was raised to a GST-CspA fusion protein expressed from E. coli (see Methods), and the antibody was used to test different cellular fractions of GBS for the presence of CspA. Periplasmic extracts (see Methods) of E. coli strain TOH50 (bearing plasmid PTH5; see above) reacted strongly with the antibody raised to GST-CspA. Plasmid PTH5 contains a mutation in the CspA coding region (in comparison with the cspA gene of the wild-type isogenic strain, $\mathrm{COH} 1$ ) that prematurely terminates translation at Leu-1121; this accounts for the lower molecular mass observed for the TOH50 extracts in comparison with wild-type CspA. Proteins from culture supernatants of $\mathrm{COH} 1$ did not react with the antibody, even when concentrated 10-fold (data not shown). In contrast, Western blots of mutanolysinextracted surface proteins from both $\mathrm{COH} 1$ and TOH97 $\left(c s p A^{+}, \operatorname{scp} B^{-}\right)$revealed two protein bands of molecular masses 142 and $80 \mathrm{kDa}$ (Figure 3). The migration of COH1-derived CspA on SDS-PAGE was anomalous, since it corresponded to a lower molecular mass (142 $\mathrm{kDa}$ ) than predicted by sequence analysis for mature CspA $(153 \mathrm{kDa})$. No cross-reactive bands were seen at the 142-kDa position for the cspA mutants (TOH121 and TOH144), confirming that the antibody does react with wild-type CspA from GBS. The results of the sequence analysis of CspA taken together with the Western blot data indicate the CspA is a surface-localized protein.

\section{Table 1}

Strains and plasmids

$\begin{array}{lll}\begin{array}{l}\text { Strain or } \\ \text { plasmid }\end{array} & \text { Relevant characteristics } & \begin{array}{l}\text { Reference } \\ \text { or source }\end{array} \\ \text { Strain } & & \end{array}$

S. agalactiae

$\mathrm{COH} 1$ Type III GBS isolate from neonate with septicemia; highly virulent; wild-type reference strain

$\mathrm{TOH} 121$

TOH97

cspA::erm derivative of $\mathrm{COH} 1 ; \mathrm{Erm}^{r}$ $\operatorname{scp} B::$ kan derivative of $\mathrm{COH} 1 ; \mathrm{Kan}^{r}$

$\mathrm{TOH} 144$ scpB::kan derivative of $\mathrm{TOH} 121$ (cspA::erm); $\mathrm{Erm}^{r}, \mathrm{Kan}^{r}$

this work 54

E. coli

$\mathrm{DH} 5 \alpha$

$\mathrm{TOH} 50$

General cloning strain

Plasmid

$\mathrm{DH} 5 \alpha$ bearing plasmid $\mathrm{pTH} 5$

ATCC

this work

PBSKS-

PVE6007

General cloning vector

E. coli-GBS shuttle vector; temperature -sensitive replication; $\mathrm{pWW}$ origin of replication; $\mathrm{Cm}^{r}$ $\begin{array}{ll}\text { PTH2 } & \text { pBSKS bearing Clal DNA fragment } \\ \text { of } \operatorname{csp} A ; \mathrm{Amp}^{r}\end{array}$

PTH5 PBSKS bearing Xbal DNA fragment containing cspA; Ampr

pTH21 of PVE6007; $\mathrm{Cm}^{\mathrm{r}} ; \mathrm{Erm}^{\mathrm{r}}$ 


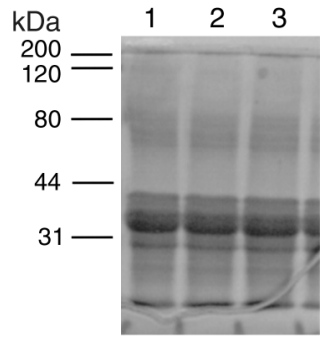

\section{Figure 5}

Caseinase activity assay. GBS strain $\mathrm{COH} 1$ was grown to stationary phase in THB, washed in PBS, concentrated 20-fold, and resuspended in PBS. A saturated solution of protease assay-grade casein (SigmaAldrich) was prepared, and $0.2 \mathrm{ml}$ of bacteria was mixed with $0.3 \mathrm{ml}$ of casein. A mock reaction with PBS (no bacteria) was also prepared. The mixtures were incubated for 24 hours at $37^{\circ} \mathrm{C}$, and an aliquot was removed for SDS-PAGE. Molecular-weight markers are depicted on the left. Lane 1 shows the PBS control, lane 2, $\mathrm{COH} 1$ after 0 hours of incubation, and lane 3, $\mathrm{COH} 1$ after 24 hours of incubation. Migration of molecular mass markers (in $\mathrm{kDa}$ ) is indicated on the left.

CspA does not function as a C5a protease. Given the strong similarity of CspA to ScpB, the C5a peptidase of GBS, we hypothesized that CspA may also be active as a C5a protease. We measured C5a-ase activity (Figure 4) with a functional assay described previously (11). Briefly, GBS was preincubated with recombinant human C5a, purified human PMNs were added, and C5a-stimulated PMN adherence to gelatin-coated plastic was measured. $\mathrm{COH} 1$ (wild-type, $s c p B^{+}$) served as a positive control, and TOH97 $\left(s c p B^{-}\right)$and another $s c p B^{-}$strain, GW (32), served as negative controls. The effect of the $\operatorname{csp} A$ mutation was measured both in the presence and absence of the GBS C5a protease (ScpB) by comparing TOH121 $\left(s c p B^{+}, c s p A^{-}\right)$and TOH144 $\left(s c p B^{-}, c s p A^{-}\right)$with the controls. Preincubation of $\mathrm{C} 5 \mathrm{a}$ with $\mathrm{COH} 1$ or TOH121 abolished C5a activity, and the C5a protease activities of the strains were similar (Figure 4). In contrast, C5a protease activity of strains GW, TOH97, and TOH144 were low and not significantly different from each other. Thus, inactivation of CspA did not appear to have a detectable effect on C5a activity, as evaluated in this functional assay. C5a protease activity only correlated with the presence of functional $\mathrm{ScpB}$. The above results, taken together, are consistent with the conclusion that CspA does not function as a C5a protease.

Evaluation of GBS phenotypic traits. We evaluated the expression of several GBS phenotypic traits, some of which are known GBS virulence determinants. The $\operatorname{csp} A$ mutant expressed $\alpha$-hemolysin, CAMP factor, hippuricase, and hyaluronidase similarly to wild-type GBS (data not shown). These strains also expressed equivalent amounts of type III capsule as measured by competitive ELISA $(72.7 \pm 15.8 \mu \mathrm{g} / \mathrm{mg}$ for $\mathrm{COH} 1$ and $82.9 \pm 26.3 \mu \mathrm{g} / \mathrm{mg}$ dry weight for TOH121). Invasion of A549 epithelial cell monolayers was $1.2 \% \pm 0.3 \%$ for $\mathrm{COH} 1$ and $1.3 \% \pm 0.2 \%$ for TOH 121 , expressed as a percentage of the number of total input bacteria invading the A549 cells.
Many of the proteases that are homologous to CspA are important for bacterial growth. For example, caseinases from lactic acid bacteria participate in the degradation of extracellular casein before utilization of the casein peptides as a nutritional source. Because GBS is auxotrophic for multiple amino acids, it must rely on exogenous amino acids. Thus, we compared the growth characteristics of the cps $A$ mutant to the wild-type strain in different growth media. Growth of the strains was comparable in all media that were tested, including RPMI plus 5\% Casamino acids and THB (data not shown). Growth in human plasma was compared, and the strains exhibited doubling times of 0.6 hours and 0.52 hours for $\mathrm{COH} 1$ and TOH121, respectively. This suggests that CspA does not play a role in nutritional scavenging, at least under the experimental conditions tested.

Caseinase activity of GBS. Because of the strong similarity of CspA to PrtS, which functions as a caseinase, we tested whole cells of COH1 and TOH121 for casein degradation. Whole GBS cells were incubated with casein, the mixture was centrifuged, SDS-PAGE was performed on the supernatant, and the amount of intact casein was measured by quantitation of Coomassie-stained gels. Very little casein degradation was observed under the experimental conditions employed, and experiments with the two strains yielded similar results (Figure 5).

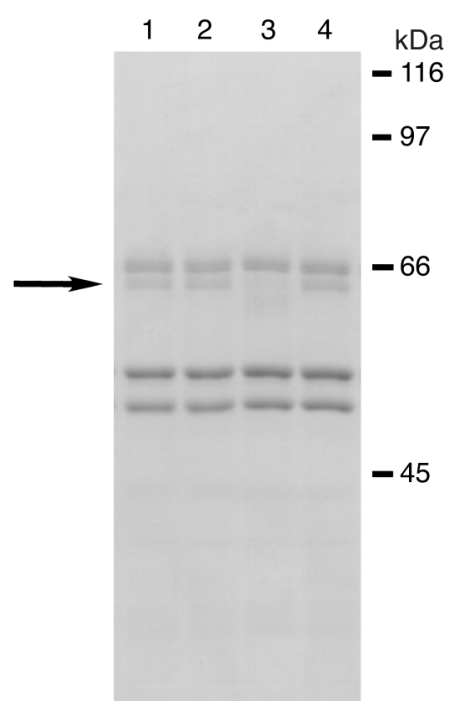

\section{Figure 6}

Fibrinogen degradation assay. GBS strains $\mathrm{COH} 1$ (wild type) and $\mathrm{TOH} 121\left(\operatorname{csp} A^{-}\right)$were grown to stationary phase in THB. Cells were washed once in an equivalent volume of PBS and concentrated 20fold. Fibrinogen was then added to a concentration of $0.61 \mu \mathrm{g} / \mu \mathrm{l}$. The suspension was incubated with slow rotation overnight (16 hours) at $37^{\circ} \mathrm{C}$, bacteria were removed by centrifugation, and the supernatant, containing $3 \mu \mathrm{g}$ of total fibrinogen, was analyzed by SDS-PAGE as follows: lane 1 shows $\mathrm{COH} 1$ after 0 hours of incubation; lane 2, TOH121 after 0 hours incubation; lane 3, $\mathrm{COH} 1$ after 16 hours of incubation; and lane 4, TOH121 after 16 hours of incubation. The arrow at the left of the figure denotes the migration position of the minor species of the $\alpha$ fragment of fibrinogen that is proteolyzed by CspA. Migration of molecular mass markers (in $\mathrm{kDa}$ ) is indicated on the right. 


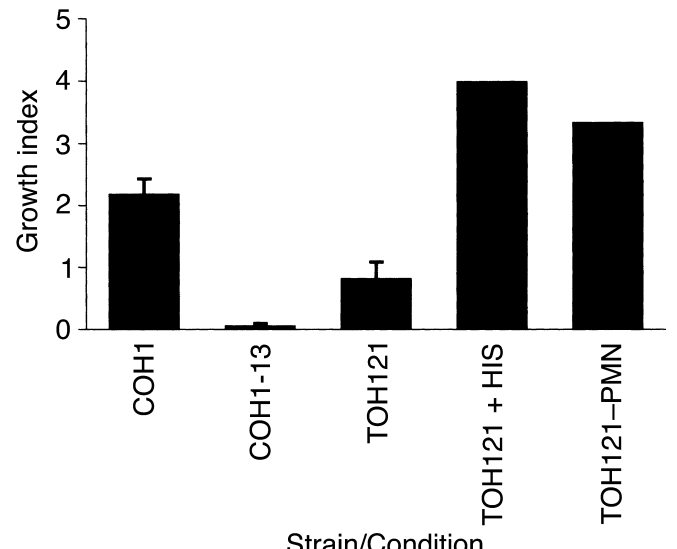

Figure 7

Opsonophagocytosis of GBS strains by PMNs. GBS strains $\mathrm{COH} 1$, $\mathrm{TOH} 121$, and the unencapsulated mutant $\mathrm{COH}$ 1-13 were compared for resistance to opsonophagocytosis by PMNs. Bacteria $\left(1 \times 10^{6}\right)$ and human PMNs $\left(3 \times 10^{6}\right)$ were incubated in $10 \%$ human sera as a complement source (preabsorbed with $\mathrm{COH} 1$ to remove $\mathrm{GBS}$ antibody) for 1 hour at $37^{\circ} \mathrm{C}$. The GI was calculated as the output CFU per milliliter divided by the input CFU per milliliter. One representative experiment of four is depicted. Controls were performed for the test strain, TOH121, with heat-inactivated serum (HIS) and without PMNs.

The cspA gene is required for cleavage of human fibrinogen. We hypothesized that CspA, as a putative surface-localized protein, proteolyses a host factor. Therefore, to test the ability of CspA to function as a protease, we compared the ability of the $\operatorname{csp} A$ mutant and the wild-type strain to degrade a variety of host proteins. We tested purified human fibronectin, purified complement component C3, and purified human fibrinogen. To evaluate degradation, whole bacteria and/or mutanolysinextracted surface proteins of $\mathrm{COH} 1$ and $\mathrm{TOH} 121$ were incubated with the test substrate, and degradation was assessed by evaluation of Coomassie-stained SDS-PAGE gels or by Western blotting. No difference in the cleavage of the test substrates was observed for all substrates except fibrinogen when the mutant and wild-type strains were compared (data not shown).

Human fibrinogen was cleaved by the wild-type strain but was not cleaved by the cspA mutant TOH121. Fibrinogen is a dimer of nonidentical subunits $-\alpha, \beta$, and $\gamma-$ that are covalently linked together by disulfide bonds (42). For fibrinogen isolated from fresh human plasma, we found that SDS-PAGE resolves the $\alpha$ subunit of fibrinogen into a doublet. We excised both putative $\alpha$ bands from an SDS-PAGE gel and confirmed their identity by MALDI-TOF MS. Peptides matching the sequence of the $\alpha$ fragment of human fibrinogen were obtained from both putative $\alpha$ bands; $28 \%$ of the fibrinogen sequence was covered by the tryptic peptides that were obtained. The lower band of the $\alpha$ doublet is believed to be an in vivo cleavage product in which the C-terminal 27 amino acids are removed (43). When the wild-type and CspA mutants were compared for fibrinogen cleavage, SDS-PAGE revealed that
$\mathrm{COH} 1$ cleaved the minor species (corresponding to the lower band of the doublet) of the $\alpha$ subunit, whereas the mutant did not cleave fibrinogen under the conditions used (Figure 6). We also noticed that when the mutant and wild-type were incubated with fibrinogen overnight, the wild-type strain formed macroscopic aggregates that were much more prominent in comparison with the mutant strain. These aggregates were not observable in controls in which the wild-type strain was incubated in PBS alone.

CspA is essential for virulence in a neonatal rat model. Extracellular proteases have been implicated in virulence both in Gram-negative and Gram-positive bacteria. To determine if CspA contributes to virulence, we compared the $\mathrm{LD}_{50}$ of TOH121 (csp $\left.A^{-}\right)$to the isogenic wild type strain, $\mathrm{COH} 1$. A series of 10 -fold dilutions of each strain were introduced by intraperitoneal injection into neonatal rats (24-48 hours old). Five separate lethality experiments were performed. The mean $\mathrm{LD}_{50}$ values were $2.9 \times 10^{3}$ and $2.9 \times 10^{4}$ CFU per animal for COH1 and TOH121, respectively (P $\leq 0.0431$ by the Wilcoxon matched-pair signedranks test). These results suggest that mutation of $\operatorname{csp} A$ significantly impairs the virulence of GBS in the neonatal sepsis model.

CspA promotes evasion of opsonophagocytosis. We hypothesized that CspA may allow GBS to avoid innate immune clearance in the nonimmune host, perhaps by a novel mechanism, since capsular polysaccharide expression was not affected by the cspA mutation (see above). Opsonophagocytosis is an important mechanism for bacterial clearance, and neutrophils play a major role in the elimination of GBS from the bloodstream (44). We hypothesized that the attenuated virulence of TOH121 may be a consequence of increased susceptibility to phagocytic clearance as compared with COH1. Fresh PMNs were isolated from human donors, and pooled human serum was used as a source of complement and was preabsorbed with $\mathrm{COH} 1$ to remove antibodies directed against the bacteria. The assay was repeated four times, and the results of a representative experiment are shown in Figure 5. COH 1-13 (16), which is an unencapsulated mutant of $\mathrm{COH} 1$ known to be very susceptible to PMN killing, was included as a control. The growth index (GI) of each strain was calculated as the output CFU per milliliter divided by the input CFU per milliliter. $\mathrm{COH} 1$ growth during the assay corresponded to more than one doubling ( $\mathrm{GI}=2.2$ ) (Figure 7). In contrast, the negative control strain, $\mathrm{COH} 1-13$, was markedly killed by human PMNs during the 1-hour incubation $(\mathrm{GI}=0.04)$. TOH121 exhibited a sensitivity that was intermediate between $\mathrm{COH} 1$ and $\mathrm{COH} 1-13(\mathrm{GI}=0.81)$. All three strains grew in the presence of heat-inactivated sera and in the absence of PMNs, yielding GIs between 2 and 4 (Figure 7 and data not shown). We also averaged the results of the four experiments and expressed the results as a ratio of the GI of the mutant to the GI of the wild type, which was 0.46 ( $P<0.001$ by Student's $t$ test). These findings suggest that CspA promotes resistance 
to nonimmune opsonization and killing by PMNs, though not to the same degree as observed for the capsular polysaccharide (16).

The cspA gene is widely distributed among the GBS serotypes. Since CspA contributes to virulence, we investigated the prevalence of $\operatorname{csp} A$ in the other GBS serotypes. Southern blots were performed using GBS genomic DNA isolated from representative strains from each of the nine serotypes. A single DNA fragment from 16 of 18 strains hybridized to the $\operatorname{csp} A$ probe (data not shown), and DNA from at least one representative of each serotype hybridized to the probe. Therefore, the $\operatorname{csp} A$ gene is prevalent among all the serotypes examined.

\section{Discussion}

It is clear that bacterial proteases have diverse functions in pathogenesis. Proteases can benefit the invading microbe by liberating nutrients from the host $(45,46)$ or can promote disease through direct destruction of host tissues (47). Several proteases activate host zymogens, such as plasminogen (48), kininogens, matrix metalloproteases, and proenzymes of the clotting system (47). Some of the proteases inactivate host-defense proteins, such as immunoglobulins, components of the complement system, and antimicrobial peptides (48-50), whereas others are required for intracellular survival in macrophages (49) and for adherence as well as uptake into epithelial cells (50).

In this study, we identified a GBS gene encoding a protein that shows strong similarity to CEPs from other Gram-positive bacteria and contributes to GBS virulence. CEPs are a branch of a larger family of proteins known as subtilases, which use a serine active site (51). The overall similarity of CspA to CEP proteins as well as the conservation of residues associated with the catalytic activity of serine proteases (aspartic acid, histidine, and serine motifs) suggest that CspA functions as a protease. For a comprehensive review of the domain structure of CEPs, see Siezen (36). The presence of a putative signal peptide and a cell-wall anchor (LPXTG) motif indicates that CspA is a cell-surface-associated protein, and Western blots of cell-surface-associated proteins confirmed the surface localization of CspA. Although CspA shares many of the features of the CEPs, including a putative pre-pro domain and anchor domain, CspA diverges from members of the CEP family in other aspects of its sequence. Two domains that are present in other CEPs, the "H" domain, thought to function as a cellwall spacer, and the "W" domain, thought to function as a cell-wall-spanning domain, are absent in CspA. These domains are replaced with a novel sequence that is not found in the other CEPs; no homologous sequences in the existing protein database were found (36). The significance of these observations is not known at present but suggest that CspA has a distinct structure from the known CEPs in the vicinity of the cell-wall-proximal region, which may also influence localization of CspA.
CspA appears to be a novel member of the CEP family of subtilase-like proteases on the basis of its functional properties also. Most of the CEP family members that have been studied are from dairy lactic acid bacteria and function in the acquisition of nutrients through degradation of casein (36). In fact, the CEP family member that CspA shows the highest overall similarity to is PrtS from S. thermophilus (12), which catalyzes casein degradation. However, the inactivation of CspA did not affect the ability of GBS to degrade casein in vitro; in fact, GBS did not exhibit substantial caseinase activity under the conditions that we tested. We also considered that CspA may function to degrade other proteins for the purpose of nutrient acquisition. However, we did not detect a difference in growth rates in human plasma when the wild-type strain and the $\operatorname{csp} A$ mutant were compared in vitro (doubling times of 0.6 hours and 0.52 hours for $\mathrm{COH} 1$ and TOH121, respectively). The findings suggest that CspA does not perform a function homologous to the caseinases (36) and does not play an essential role in nutrient acquisition, at least under the conditions that we tested.

The finding that whole cells of TOH121 (csp $\left.A^{-}\right)$cannot cleave fibrinogen strongly suggests that $\operatorname{csp} A$ encodes a protein with active proteolytic activity. Although it is a formal possibility that the $\operatorname{csp} A$ gene is simply necessary for the function of a distinct protease that actually cleaves fibrinogen, the simplest hypothesis to explain our data is that $\operatorname{csp} A$ encodes the fibrinogen-degrading protease. It is intriguing that CspA degrades the lower $\alpha$ band of fibrinogen, which corresponds to fibrinogen with the C-terminal 29 amino acids removed (43). Many proteases, including plasmin, will cleave the $C$ terminus of fibrinogen (43), but it appears that CspA acts on the form of fibrinogen that has already been cleaved at the C terminus of the $\alpha$ chain. The significance of this observation is not currently known; however, CspA may proteolyse fibrinogen at a position distinct from the positions acted on by other bacterial proteases.

Another bacterial protease that degrades fibrinogen is the SpeB protease of GAS. SpeB also cleaves fibrinogen, but in a manner that is rather different from what we observed for CspA activity. SpeB degrades the $\alpha$ band of fibrinogen, which leads to the nearly complete disappearance of the $\alpha$ band on SDS-PAGE gels (52). In contrast, CspA does not degrade the major species of the $\alpha$ chain, and its cleavage of the minor $\alpha$ species yields bands that differ in size from the major band by less than $10 \mathrm{kDa}$. These results suggest that CspA may cleave fibrinogen more specifically than SpeB; additionally, SpeB is a cysteine protease, whereas CspA is a serine protease.

The connection between the attenuated opsonophagocytosis, the reduced virulence of TOH121, and the fibrinolytic activity of CspA is not yet clear. However, the sensitivity of TOH121 to opsonophagocytic killing may reflect the inability of TOH121 to cleave fibrinogen. GBS may be exploiting the adhesive properties of a fibrin-like substance by cleaving host fibrinogen in a manner sim- 
ilar to thrombin. Fibrin is formed from fibrinogen and is the key structural element of blood clots as well as a component of the extracellular matrix. In blood, the proteolysis of the $\mathrm{N}$ termini of the $\alpha$ and $\beta$ chains of fibrinogen leads to the polymerization of cleaved fibrinogen, forming fibrin, which, as the structural component of blood clots, is highly adhesive. If CspA cleaves the $\alpha$ chain of fibrinogen at the $\mathrm{N}$ terminus, it could potentially expose the charged regions present on fibrinogen that are responsible for polymerization of fibrinogen and may promote the aggregation of GBS or the coating of GBS with fibrin. We have observed that when the wild-type strain (COH1) is incubated with fibrinogen, aggregates are formed and can be observed as macroscopic particles, whereas in the TOH121 (csp $\left.A^{-}\right)$mutant strain, aggregation is much less prominent. These aggregates may represent GBS complexed with a fibrinogenderived cleavage product.

One explanation for the reduced opsonophagocytosis of TOH121 is that wild-type GBS $\left(\operatorname{csp} A^{+}\right)$is coated with a fibrin-like substance and this substance reduces the access of opsonins or neutrophils to GBS. The other possibility is that the cellular aggregation is induced by fibrinogen cleavage; aggregated GBS may be protected from host-defense mechanisms. A third possibility is that the reduced opsonophagocytosis of GBS is not a consequence of CspA-cleaved fibrinogen. For example, it is possible that CspA proteolyses a protein distinct from fibrinogen and that degradation of this protein is responsible for the attenuated opsonophagocytosis of TOH121. Such a protein could even be bacterial in nature; the SpeB protease of GAS is known to cleave bacterial surface proteins (52). We tested for degradation of the GBS $\alpha \mathrm{C}$ protein and found that the cspA gene was not required for cleavage of this surface-localized protein (data not shown). Further studies will be needed in order to link the defect in virulence to the attenuated opsonophagocytosis and fibrinogen cleavage.

We attempted to complement the cspA mutant in order to verify that that decreased opsonophagocytosis, virulence, and defect in fibrinogen cleavage were linked. However, we were unable to subclone $\operatorname{csp} A$ to a plasmid; attempts at cloning on both high- and low-copy plasmids failed. We are currently attempting to replace the $\mathrm{erm}^{\mathrm{r}}$ cassette of TOH121 with the wild-type gene in order to demonstrate the causality of the $\operatorname{csp} A:: \mathrm{erm}^{\mathrm{r}}$ mutation. Gram-positive bacteria are important human pathogens; however, much remains to be understood concerning how Gram-positive bacteria evade opsonophagocytosis. To our knowledge, this study represents the first report of a GBS protease that functions in the evasion of opsonophagocytosis. In summary, we have identified a novel GBS protease with extensive homology to the C5a-ases of GAS and GBS as well as to other members of the subtilase family of serine proteases. All GBS serotypes that were examined bore the $\operatorname{cs} p A$ gene. Mutation of $\operatorname{csp} A$ attenuated GBS virulence in a neonatal rat sepsis model and decreased resistance to phagocytosis. The protease is necessary for the cleavage of human fibrinogen. Understanding the contribution of this novel serine protease-like gene to the pathogenesis of GBS disease will broaden our understanding of how this significant pathogen causes severe infections of the newborn infant.

\section{Acknowledgments}

We thank Donald Chaffin, Jason Short, Clementien Vermont, and Peter Vickerman for their technical assistance with this project as well as Lakshmi Rajagopal for the accompanying scanning micrograph. We also thank Roland Siezen and Glen Tamura for their advice. This work was supported by NIH grants AI30068 (C. E. Rubens) and RO1AI 40918 (J.F. Bohnsack) and by National Research Service Award 5 F32 AI10426-03 (D. W. Shelver).

1. Baker, C.J., and Edwards, M.S. 1995. Group B Streptococcal infections. In Infectious diseases of the fetus and newborn infant. J.S. Remington and J.O. Klein, editors. W. B. Saunders. Philadelphia, Pennsylvania, USA. 980-1054.

2. Farley, M.M., et al. 1993. A population-based assessment of invasive disease due to group B streptococcus in nonpregnant adults. N. Engl. J. Med. 328:1807-1811.

3. Ernst, J. 2000. Bacterial inhibition of phagocytosis. Cell. Microbiol. 2:379-386.

4. Dillard, J.P., Vandeersea, M.W., and Yother, J. 1995. Characterization of the cassette containing genes for type 3 capsular polysaccharide biosynthesis in Streptococcus pneumoniae. J. Exp. Med. 181:973-983.

5. Cheng, Q., Finkel, D., and Hostetter, M.K. 2000. Novel purification scheme and functions for a C3-binding protein from Streptococcus pneumoniae. Biochemistry. 39:5450-5457.

6. Hancock, L.E., and Gilmore, M.S. 2002. The capsular polysaccharide of Enterococcus faecalis and its relationship to other polysaccharides in the cell wall. Proc. Natl. Acad. Sci. USA. 99:1574-1579.

7. Ashbaugh, C., Warren, H.B., Carey, V.J., and Wessels, M.R. 1998. Molecular analysis of the role of the group A streptococcal cystein protease, hyaluronic acid capsule, and $\mathrm{M}$ protein in a murine model of human invasive soft-tissue infection. J. Clin. Invest. 102:550-560.

8. Wessels, M.R., Moses, A.E., Goldberg, J.B., and DiCesare, T.J. 1991. Hyaluronic acid capsule is a virulence factor for mucoid group A streptococci. Proc. Natl. Acad. Sci. USA. 88:8317-8321.

9. Rubens, C.E., Wessels, M.R., Heggen, L.M., and Kasper, D.L. 1987. Transposon mutagenesis of type III group B Streptococcus: correlation of capsule expression with virulence. Proc. Natl. Acad. Sci. USA. 84:7208-7212.

10. Areschoug, T., Stalhammar-Carlemalm, M., Karlsson, I., and Lindahl, G. 2002. Streptococcal $\beta$ protein has separate binding sites for human factor $\mathrm{H}$ and IgA-Fc. J. Biol. Chem. 277:12642-12648.

11. Bohnsack, J.F., et al. 1991. Purification of the proteinase from Group B Streptococci that inactivates human C5a. Biochim. Biophys. Acta. 1079:222-228

12. Fernandez-Espla, M.D., Garault, P., Monnet, V., and Rul, F. 2000. Streptococcus thermophilus cell wall-anchored proteinase: release, purification, and biochemical and genetic characterization. Appl. Environ. Microbiol. 66:4772-4778.

13. Martin, T.R., Rubens, C.E., and Wilson, C.B. 1988. Lung antibacterial defense mechanisms in infant and adult rats: implications for the pathogenesis of Group B Streptococcal infections in the neonatal lung. J. Infect. Dis. 157:91-100.

14. Tamura, G.S., and Rubens, C.E. 1995. Group B streptococci adhere to a variant of fibronectin attached to a solid phase. Mol. Microbiol. 15:581-589.

15. Chmouryguina, I., Suvorov, A., Ferrieri, P., and Cleary, P.P. 1996. Conservation of the C5a peptidase genes in Group A and B Streptococci. Infect. Immun. 64:2387-2390.

16. Rubens, C.E., Heggen, L.M., Haft, R.F., and Wessels, M.R. 1993. Identification of cpsD, a gene essential for type III capsule expression in Group B Streptococci. Mol. Microbiol. 8:843-855.

17. Sambrook, J.E., Fritsch, F., and Maniatis, T. 1989. Molecular cloning: a laboratory manual. Cold Spring Harbor Laboratory Press. Cold Spring Harbor, New York, USA. pp. 1659.

18. Yim, H.H., and Rubens, C.E. 1997. Use of a dental amalgamator to extract RNA from the gram-positive bacterium Streptococcus agalactiae. Biotechniques. 23:229-231.

19. Maguin, E., Duwat, P., Hege, T., Ehrlich, D., and Gruss, A. 1992. New 
thermosensitive plasmid for gram-positive bacteria. J. Bacteriol. 174:5633-5638.

20. Rubens, C.E., and Heggen, L.M. 1988. Tn916 delta E: a Tn916 transposon derivative expressing erythromycin resistance. Plasmid. 20:137-142.

21. Framson, P.E., Nittayajarn, A., Merry, J., Youngman, P., and Rubens, C.E. 1997. New genetic techniques for Group B Streptococci: high-efficiency transformation, maintenance of temperature-sensitive $\mathrm{pWV01}$ plasmids, and mutagenesis with Tn917. Appl. Environ. Microbiol. 63:3539-3547.

22. Yim, H.H., and Rubens, C.E. 1998. Site-specific homologous recombination mutagenesis in group B streptococci. Methods. Cell. Sci. 20:13-20.

23. Chaffin, D.O., Beres, S.B., Yim, H.H., and Rubens, C.E. 2000. The serotype of type Ia and III Group B Streptococci is determined by the polymerase gene within the polycistronic capsule operon. J. Bacteriol. 182:4466-4477.

24. Nizet, V., et al. 1996. Group B streptococcal beta-hemolysin expression is associated with injury of lung epithelial cells. Infect. Immun. 64:3818-3826.

25. Richman, P.G., and Baer, H. 1980. A convenient plate assay for the quantitation of hyaluronidase in Hymenoptera venoms. Anal. Biochem. 109:376-381.

26. Ferrieri, P., Wannamaker, L.W., and Nelson, J. 1973. Localization and characterization of the hippuricase activity of group B streptococci. Infect. Immun. 7:747-752.

27. Winram, S.B., Tamura, G.S., and Rubens, C.E. 1998. In vitro systems for investigating group B streptococcal host cell and extracellular matrix interactions. Methods. Cell. Sci. 20:191-201.

28. Bohnsack, J.F., Mollison, K.W., Buko, A.M., Ashworth, J.C., and Hill, H.R 1991. Group B streptococci inactivate complement component C5a by enzymic cleavage at the C-terminus. Biochem. J. 273:635-640.

29. Jones, A.L., Knoll, K.M., and Rubens, C.E. 2000. Identification of Streptococcus agalactiae virulence genes in the neonatal rat sepsis model using signature-tagged mutagenesis. Mol. Microbiol. 37:1444-1455.

30. Harlow, E., and Lane, D. 1988. Antibodies: a laboratory manual. Cold Spring Harbor Laboratory Press. Cold Spring Harbor, New York, USA. 68 pP.

31. Tanaka, T., and Weisblum, B. 1975. Construction of a colicin E1-R factor composite plasmid in vitro: means for amplification of deoxyribonucleic acid. J. Bacteriol. 121:354-362.

32. Bohnsack, J.F., et al. 2000. Genetic polymorphisms of group B streptococcus scpB alter functional activity of a cell-associated peptidase that inactivates C5a. Infect. Immun. 68:5018-5025.

33. Baltimore, R.S., Kasper, D.L., Baker, C.J., and Goroff, D.K. 1977. Antigenic specificity of opsonophagocytic antibodies in rabbit anti- sera to Group B Streptococci. J. Immunol. 118:673-678.

34. Ferretti, J.J., et al. 2001. Complete genome sequence of an M1 strain of Streptococcus pyogenes. Proc. Natl. Acad. Sci. USA. 98:4658-4663.

35. Chen, C.C., and Cleary, P.P. 1989. Cloning and expression of the streptococcal C5a peptidase gene in Escherichia coli: linkage to the type $12 \mathrm{M}$ protein gene. Infect. Immun. 57:1740-1745.

36. Siezen, R.J. 1999. Multi-domain, cell-envelope proteinases of lactic acid bacteria. Antonie Van Leeuwenhoek. 76:139-155.
37. White, O., et al. 1999. Genome sequence of the radioresistant bacterium Deinococcus radiodurans $\mathrm{R} 1$. Science. 286:1571-1577.

38. Belitsky, B.R., Gustafsson, M.C., Sonenshein, A.L., and Von Wachenfeldt, C. 1997. An lrp-like gene of Bacillus subtilis involved in branched-chain amino acid transport. J. Bacteriol. 179:5448-5457.

39. Stock, J.B., Ninfa, A.J., and Stock, A.M. 1989. Protein phosphorylation and regulation of adaptive responses in bacteria. Microbiol. Rev. 53:450-490.

40. von Heijne, G. 1986. A new method for predicting signal sequence cleavage sites. Nucleic. Acid. Res. 14:4683-4690.

41. Navarre, W.W., and Schneewind, O. 1994. Proteolytic cleavage and cell wall anchoring at the LPXTG motif of surface proteins in gram-positive bacteria. Mol. Microbiol. 14:115-121.

42. Doolittle, R.F. 1984. Fibrinogen and fibrin. Annu. Rev. Biochem. 53:195-224.

43. Cottrell, B.A., and Doolittle, R.F. 1976. The amino acid sequence of a 27residue peptide released from the alpha-chain carboxy-terminus during the plasmic digestion of human fibrinogen. Biochem. Biophys. Res. Commun. 71:754-761.

44. Nizet, V., Ferrieri, P., and Rubens, C.E. 2000. Molecular pathogenesis of group B streptococcal disease in newborns. In Streptococcal infections: clinical aspects, microbiology, and molecular pathogenesis. Oxford University Press. New York, New York, USA. pp.180-221.

45. Lantz, M.S. 1997. Are bacterial proteases important virulence factors? J. Periodontal Res. 32:126-132.

46. Projan, S.J., and Novick, R.P. 1997. The molecular basis of pathogenicity. Churchill Livingstone. New York, New York, USA. pp. 55-81.

47. Maeda, H., and Yamamoto, T. 1996. Pathogenic mechanisms induced by microbial proteases in microbial infections. Biol. Chem. Hoppe. Seyler. 377:217-226

48. Lähteenmäkia, K., Pentti, K., and Korhonen, T.K. 2001. Bacterial plasminogen activators and receptors. FEMS Microbiol. Rev. 25:531-555.

49. Gaillot, O., Pellegrini, E., Bregenholt, S., Nair, S., and Berche, P. 2000. The $\mathrm{ClpP}$ serine protease is essential for the intracellular parasitism and virulence of Listeria monocytogenes. Mol. Microbiol. 35:1286-1294.

50. St. Geme, J.W., III, and Cutter, D. 1995. Evidence that surface fibrils expressed by Haemophilus influenzae type b promote attachment to human epithelial cells. Mol. Microbiol. 15:77-85.

51. Siezen, R.J., and Leunissen, J.A. 1997. Subtilases: the superfamily of subtilisin-like serine proteases. Protein. Sci. 6:501-523.

52. Matsuka, Y., Pillai, S., Gubba, S., Musser, J.M., and Olmsted, S.B. 1999. Fibrinogen cleavage by the Streptococcus pyogenes extracellular cysteine protease and generation of antibodies that inhibit proteolytic activity. Infect. Immun. 67:4326-4333.

53. D’Souza, S.E., Ginsberg, M.H., and Plow, E.F. 1991. Arginyl-glycyl-aspartic acid (RGD): a cell adhesion motif. Trends. Biochem. Sci. 16:246-250.

54. Beckmann, C., Waggoner, J.D., Harris, T.O., Tamura, G.S., and Rubens, C.E. 2002. Identification of novel adhesins from Group B Streptococci by use of phage display reveals that C5a peptidase mediates fibronectin binding. Infect. Immun. 70:2869-2876. 